\section{An attempt to induce transient immunosuppression pre-ery- throcytapheresis in a girl with sickle cell disease, a history of severe delayed hemolytic transfusion reactions and need for hip prosthesis}

\author{
Alessandro Cattoni, ${ }^{1}$ \\ Giovanni Cazzaniga, ${ }^{2}$ Paolo Perseghin, ${ }^{3}$ \\ Giovanni Zatti, ${ }^{4}$ Diego Gaddi, ${ }^{4}$ \\ Andrea Cossio, ${ }^{4}$ Andrea Biondi, ${ }^{1}$ \\ Paola Corti, ${ }^{1}$ Nicoletta Masera ${ }^{1}$ \\ 1Pediatric Department, \\ ${ }^{2}$ Immunotransfusional Service, \\ ${ }^{3}$ Apheresis Unit, ${ }^{4}$ Orthopedic \\ Department, San Gerardo Hospital, \\ Monza, Italy
}

\section{Abstract}

We report on a case of delayed hemolytic transfusion reaction (DHTR) occurred 7 days after an erythrocytapheresis or eritroexchange procedure (EEX) treated with rituximab and glucocorticoids in a 15-years old patient with sickle cell disease. EEX was performed despite a previous diagnosis of alloimmunization, in order to reduce hemoglobin $\mathrm{S}$ rate before a major surgery for avascular necrosis of the femoral head. A first dose of rituximab was administered before EEX. However, rituximab couldn't prevent DHTR that occurred with acute hemolysis, hemoglobinuria and hyperbilirubinemia. A further dose of rituximab and three boli of methylprednisolone were given after the onset of the reaction. It is likely that the combined use of rituximab and steroids managed to gradually improve both patient's general conditions and hemoglobin levels. Nor early or late side effects were registered in a 33 -months follow-up period. This report suggests the potential effectiveness and safety of rituximab in combination with steroids in managing and mitigating the symptoms of delayed post-transfusional hemolytic reactions in alloimmunized patients affected by sickle cell disease with absolute need for erythrocytapheresis.

\section{Introduction}

The incidence of alloimmunization against red blood cells (RBC) antigens has been reported in up to $5-36 \%$ patients affected by sickle cell disease (SCD) after at least one transfusion. ${ }^{1}$ Delayed hemolytic transfusion reaction/hyperhemolysis (DHTR/H) syndrome is a complication of alloimmunization, occurring in about $11 \%$ of patients with $\mathrm{RBC}$ alloantibodies. $^{2}$

Sometimes, in particularly severe cases, this reaction constitutes a contra-indication to further transfusions. However, the use of EEX for the preparation of SCD patients to major surgery is suggested in order to reduce the short and medium-term risks and to guarantee a better outcome from surgery itself, ${ }^{3-5}$ even if this indication isn't strictly accepted by all authors and guidelines. Rituximab is a wellknown anti-CD20 immunosuppressive drug used for the treatment of autoimmune hemolytic anemias. ${ }^{6}$ The use of Rituximab in the treatment of alloimmunization-related hemolysis has been previously reported in some cases. ${ }^{7-10}$

\section{Case Report}

A 15 years old girl came to our clinic with a diagnosis of homozygous SCD. Prior to this, the patient had been sporadically transfused for episodes of severe anemia in her home country, Ghana. At the moment of her arrival in Italy, a positive direct Coombs test and a negative indirect Coombs test were detected. A first diagnosis of alloimmunization was made at the age of 10 years (in 2004), after repeated acute post-transfusional hemolytic episodes. The result of both direct and indirect Coombs tests (checked again after these events) was now positive. Anti-E and anti-S antibodies were isolated from the patient serum.

Due to the several complications the girl had developed since her arrival to our clinic, (of which, one ischemic stroke) chronic transfusions were strongly recommended. Nevertheless, considering the high risk of severe hemolysis contra-indicating transfusions, the girl was treated with hydroxiurea and erythropoietin in order to maintain good hemoglobin levels and good control of pain crises. In 2008, both direct and indirect Coombs test were negative, probably as a consequence of a drop of antibodies titer below detectable levels. Due to the presence of progressive ischemic areas in the brain as detected by magnetic resonance imaging (MRI), the attending physician decided to start a program of periodic EEX. The first procedure was apparently well tolerated. However, after 7 days, a severe DHTR/H presented with diffuse pain, worsening of clinical conditions and acute hemolytic anemia ( $\mathrm{Hb}$ nadir: $4.3 \mathrm{~g} / \mathrm{dL}$ ); the event was efficiently treated with methylprednisolone (10 mg/kg/die) and the EEX program was discontinued.

In the meantime the patient developed a
Correspondence: Nicoletta Masera, Pediatric Department, University of Milano-Bicocca, San Gerardo Hospital, via Pergolesi 33, 20052, Monza (MB), Italy.

Tel. +39.039.2333513 - Fax: +39.039 .2301646 .

E-mail: n.masera@hsgerardo.org

Key words: erythrocytapheresis, rituximab, alloimunization, avascular necrosis of femoral head, sickle cell disesase.

Contributions: the authors contributed equally.

Conflict of interests: the authors declare no potential conflict of interests.

Received for publication: 12 March 2013.

Revision received: 19 May 2013.

Accepted for publication: 21 June 2013.

This work is licensed under a Creative Commons Attribution NonCommercial 3.0 License (CC BYNC 3.0).

(C) Copyright A. Cattoni et al., 2013

Licensee PAGEPress, Italy

Hematology Reports 2013; 5:e11

doi:10.4081/hr.2013.e11

femoral head avascular necrosis on the right side. Two years later also the left femoral head was affected by osteonecrosis. At this time she had an Harris Hip score of 18.55 (<70: poor) on the right side and of 83.05 on the left side: she was unable to walk with crutches and even using a wheelchair was uncomfortable. Therefore, surgical treatment of total right hip arthroplasty became necessary.

$\mathrm{HbS}$ levels higher than $80 \%$ (representing a high risk for sickling) in the absence of anemia (Hb about $10 \mathrm{~g} / \mathrm{dL}$ ) didn't allow clinicians to recommend major surgery without a preventive EEX. Nevertheless, the high risk for DHTR represented a life threat for the girl during surgery and in the immediate post-operative phase. At this time, the direct Coombs test was still negative (same was the eluate testing), while indirect Coombs test was now positive and anti-S antibodies were detected in serum. Due to the very low titer of these antibodies, plasmapheresis was not taken into consideration. Before performing EEX, the patient's RBC phenotype was further investigated according to the main red blood cells' antigens groups (MNS, Duffy, RH, Kidd and Kell) in order to select donors with the highest possible compatibility. With regard to the Duffy system, the patient was both Fy $a$ and Fy $b$ negative; the blood units chosen were Fy a negative, in consideration of the higher probability of eliciting immunologic responses with this antigen than with $F y$ b. Although anti-E antibodies (previously isolated in serum) weren't identified at this further cross-match screening, RBC units 
selected were also antigen-E negative, in consideration of the likely fall of their titer below detectable levels at the moment of recheck; if not respected, this anamnestic response could have involved a severe hemolytic reaction.

A single dose of Rituximab $\left(375 \mathrm{mg} / \mathrm{m}^{2}\right)$ was administered i.v. four days before EEX which was performed the day before surgery (under a regimen of close clinical monitoring), obtaining $\mathrm{Hb}$ : $10 \mathrm{~g} / \mathrm{dL}$ and $\mathrm{HbS}<30 \%$ (5 units of RBC purified from SAG-mannitolo were employed). Blood Immunoglobulines (Ig) and CD20+ lymphocytes were normal before the administration of Rituximab; checked about 10 days after the first dose, the absolute concentration of $\mathrm{CD}^{+} 0^{+}$cells had dropped to 0 elements/mL (0\%), confirming its effectiveness. No clinical problems occurred in the first few days after surgery (negative hemolysis indexes, no hemoglobinuria, $\mathrm{Hb}$ : $7.2 \mathrm{~g} / \mathrm{dL}$ as expected for surgical blood loss in the first day, Hb levels stably around $7 \mathrm{~g} / \mathrm{dL}$ in the next five days). Seven days after EEX, a massive hemolytic crisis occurred (Hb: $4.7 \mathrm{~g} / \mathrm{dL}$, total bilirubin: 2.7 mg/dL, LDH: 1191, severe haemoglobinuria). Direct Coombs test was checked at this point and it was still negative. A dose of methylprednisolone was administered i.v. ( $25 \mathrm{mg} / \mathrm{kg}$ ) and a RBC transfusion was performed; the blood unit was completely hemolyzed (in spite of the best apparent compatibility at crossmatch) and hyperhemolysis was detected in the following hours (nadir $\mathrm{Hb}: 3.7 \mathrm{~g} / \mathrm{dL}$; LDH: $3875 \mathrm{mg} / \mathrm{dL}$, total bilirubin: $3.5 \mathrm{mg} / \mathrm{dL}$ ). The patient's clinical conditions and vital signs remained stable. At this stage a second dose of Rituximab (375 $\mathrm{mg} / \mathrm{m}^{2}$ ) was administered, followed by two further boli of methylprednisolone, while a strong and definite contraindication to further transfusions became mandatory (except in case of possible circulatory failure). Hemoglobin level spontaneously reached basal values and the girl was discharged after four weeks (Figure 1). Surgical or medical complication did not occur. No infections were detected and the patient did not need therapy with i.v. immunoglobulines, since serum Ig remained in the normal range for age. The blood concentration of CD20+ lymphocytes was checked six months after discharge and it was normal. The partial difference between $\mathrm{CD} 20^{+}$recovery time we've recorded and the average time described in literature in case of autoimmune haemolytic anemias (AIHA), ${ }^{11,12}$ probably depends on the shorter administration regimen we've employed: $\mathrm{CD}^{2} 0^{+}$depletion is described to last up to 9-12 months after the administration of 4 weekly doses of rituximab $\left(375 \mathrm{mg} / \mathrm{m}^{2}\right)$ in the treatment of AIHA. In the present case, only two doses were administered, and it explains the shorter duration of $\mathrm{CD}^{-} 20^{+}$lymphocytes depletion. The patient rapidly acquired complete autonomy in movement and adequate pain control.

Currently, hemoglobin levels range between $8.5 \mathrm{~g} / \mathrm{dL}$ and $9.5 \mathrm{~g} / \mathrm{dL}$ with the only use of Hydroxyurea $(25 \mathrm{mg} / \mathrm{kg} / \mathrm{die})$. From the orthopedic point of view good-excellent results were scored in the immediate postoperative time and at 1 year follow-up. No surgical site complication, no rehabilitation problems and no articular limitations appeared. At 1 year follow up no X-rays complications were evident. The Harris Hip Score reached excellent results (95.7 with the following score references: $<70$ poor; 70-79 fair; 80-89 good; >90 excellent).

\section{Discussion}

After transfusion, the exposure to alloantigens expressed on donor's RBC may lead recipient's B-lymphocytes to produce specific alloantibodies. Patients with SCD are frequently subject to repeated transfusions and present higher rates of alloimmunization than other groups treated with multiple transfusions: one of the postulated reasons is that donors and recipients often belong to different ethnical groups. Besides, RBC in SCD patients often express higher levels of phosphatydilserine, that increases both antibodies and complement fixation. ${ }^{13}$ An accurate cross-matching testing represents the most effective way to prevent alloimmunization. Nevertheless, although alloantibodies are easily isolated in serum at the beginning of the process, their concentration often falls below detectable levels throughout time: this is the reason why historical antibodies always have to be taken into consideration and honored in selecting RBC units to transfuse. Furthermore, many rare antigens can't be detected by cross-matching tests, increasing the risk of alloimmunization. A single exposure to alloantigens represents the first trigger for humoral immunity. Subsequent exposures involve B-memory cells, with much more effective and severe responses, such as DHTR. For all these reasons, alloimunized patients shouldn't be transfused as long as it is possible. However, transfusional therapy becomes sometimes mandatory, as shown in the case reported here.

Literature suggests the central role of EEX in the preparation of SCD patients to major surgery in order to reduce intra- and post-operatory risks linked to dehydration and acidosis that might in turn induce RBC sickling. ${ }^{3-5}$ EEX immediately before surgery allows the execution even of major surgical procedures in safety, by lowering $\mathrm{HbS}$ to values $<30 \%$. Alloimmunization and the consequent contraindication to transfusion represents a big issue which has to be faced in these situations. A prophylactic therapy with immunedepressive drugs becomes necessary before
EEX, in order to prevent DHTR, which is likely to occur. We describe the use of rituximab in achieving this goal in SCD.

As far as we know, there are no trials supporting this indication. Rituximab has been used in several cases to treat ongoing hyperhemolysis, ${ }^{7,8,10}$ but in only one SCD patient this therapy was reported as effective when administered before EEX preventing acute hemolytic complications. $^{9}$

The rationale behind the use of rituximab to prevent DHTR is based on a wide experience with this drug in auto-immune anemias. It both prevents the development of auto-reactive antibodies and reduces antibody-dependent cellular cytotoxicity towards RBC, by binding Fc receptor-expressing effector cells. ${ }^{14}$ Rituximab might have a dual role in preventing DHTR: i) by reducing alloantibodies production with the same mechanism described for autoantibodies and ii) by preventing hyperhemolysis. Hyperhemolisis is a separate clinical entity occurring together with DHTR as a consequence of the so-called by-stander hemolysis. This involves autologous RBC and explains the drop of hemoglobin below the levels before transfusion. ${ }^{15}$ In fact after transfusion, alloantibodies can trigger the development of autoantibodies.

The present report points out the poor reliability of both direct and indirect Coombs test in managing a transfusional program in alloimmunized patients. Even after a long time from previous transfusions and despite a negative result of these tests, alloantibodies are constantly released in serum but frequently below detectable levels. RBC transfusions should be always avoided except when they become absolutely necessary.

A single dose of rituximab didn't prove to be sufficient in the prevention of DHTR in our patient: 7 days after EEX, a massive and sudden fall in hemoglobin levels occurred (nadir: $3.7 \mathrm{~g} / \mathrm{dL}$ ) while hemolytic indexes increased; vital signs and clinical conditions remained stable and hemoglobinuria was detected. The best therapeutic regimen for the use of rituximab has yet to be established: in the case here presented, a single dose was administered before EEX, but probably a second dose, just after the procedure, could have prevented the hemolysis we observed. In fact, in a similar case reported by Noizat and Pirenne two doses of Rituximab (one administered before surgery and the other a few days later) avoided hemolysis in a similar situation. ${ }^{9}$ Anyway, rituximab probably played a central role (in association with steroid boli) in rapidly improving patient's general conditions and hemoglobin levels. No clinical trials have demonstrated that the combined administration of rituximab and corticosteroids is more effective than rituximab alone in treating AIHA. The partial result we've achieved may depend both on the 


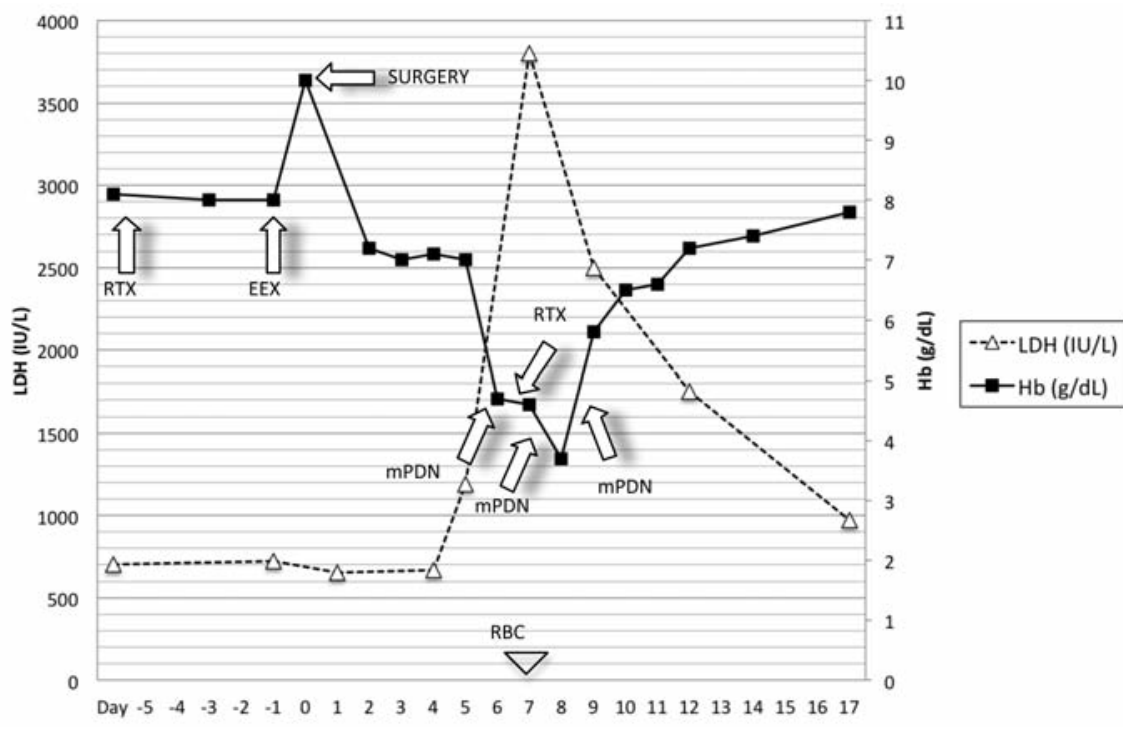

Figure 1. Trend of hemoglobin and LDH during the observation period (RBC, red blood cell transfusion; EEX, eritroexchange; RTX, rituximab; mPDN, methylprednisolone).

association of the first dose of rituximab and three boli of methyprednisolon and on the administration of a second dose of anti-CD20 about 6 days after surgery. It is possible that the administration of a second dose of rituximab immediately after surgery could effectively prevent DHTR also without corticosteroids.

The patient didn't develop any side effect and didn't need i.v. Ig treatment. We also used boli of methylprednisolone during acute hyperhemolysis without any side effect. This suggests their possible effective use in case of DHTR. Considering the high risk of infections in SCD patients and in relation to major surgery, this treatment must be used only when the history of DHTR/H is highly significant, strictly monitoring any infective complication.

\section{Conclusions}

In a patient affected by SCD presenting with alloimmunization, a history of severe episodes of DHTR/H and absolute need for EEX, rituximab showed to be a safe treatment even in association with boli of methylprednisolone in order to manage and mitigate the symptoms of delayed post-transfusional hemolytic reac- tions. Considering the partial result achieved, we suggest the administration of two doses of rituximab before transfusion, that (differently from the case here described), could even prevent DHTR to occur.

\section{References}

1. Rosse W, Gallagher D, Kinney T, et al. Transfusion and alloimmunization in sickle cell disease. Blood 1990;76:1431-7.

2. Talano JAM, Hillery CA, Gottschall JL, et al. Delayed hemolytic transfusion reaction/hyperhemolysis syndrome in children with sickle cell disease. Pediatrics 2003;111:e661.

3. Bhattacharyya N, Wayne AS, Kevy SV, Shamberger RC. Perioperative management for cholecystectomy in sickle cell disease. J Pediatr Surg 1993;28:72-5.

4. Vichinsky EP, Haberkern CM, Neumayr L, et al. A comparison of conservative and aggressive transfusion regimens in the perioperative management of sickle cell disease. The Preoperative Transfusion in Sickle Cell Disease Study Group. N Engl J Med 1995;27:206-13.

5. Associazione Italiana Ematologia
Oncologia Pediatrica (AIEOP). Raccomandazioni per la gestione della malattia drepanocitica in Italia. 2012. Available from: http:/www.aieop.org/files/ files_htmlarea/pubblicazioni_tiziana/GL/g lobulo_rosso/documenti/14.02.2011\%20rac comandazioni\%20drepanocitosi\%20Russo \%20rev.\%201.pdf. Accessed on: June 2013.

6. Zecca M, Nobili B, Ramenghi U, et al. Rituximab for the treatment of refractory autoimmune hemolytic anemia in children. Blood 2003;101:3857-61.

7. Sigler E, Shvidel L, Yahalom V, et al. Clinical significance of serologic markers related to red blood cell autoantibodies production after red blood cell transfusion - severe autoimmune hemolytic anemia occurring after transfusion and alloimmunization: successful treatment with rituximab. Transfusion 2009;49:1370-4.

8. Bley C, Aravechia MG, Hamerschlak N, et al. Autoantibody formation after alloimmunization inducing bystander immune hemolysis. Immunohematology 2009;25:912.

9. Noizat-Pirenne F, Bachir D, Chadebech P, et al. Rituximab for prevention of delayed hemolytic transfusion reaction in sickle cell disease. Haematologica 2007;92:132-5.

10. Behmeyer. Rituximab as an effective treatment of hyperhemolysis syndrome in sickle cell anemia. Am J Hematol 2010;85:91-2.

11. Stübgen JP. B cell-targeted therapy with rituximab and autoimmune neuromuscular disorders. J Neuroimmunol 2008;204:112.

12. Kumar S, Benseler SM, Kirby-Allen M, Silverman E. B-cell depletion for autoimmune thrombocytopenia and autoimmune hemolytic anemia in pediatric systemic lupus erythematosus. Pediatrics 2009;123:159.

13. Wang RH, Phillips G, Medof ME, Mold C. Activation of the alternative complement pathway by exposure of phosphatidylethanolamine and phosphatidylserine on erythrocytes from sickle cell disease patients. J Clin Invest 1993;92:1326-5.

14. Taylor RP, Lindorfer MA. Drug insight: the mechanism of action of rituximab in autoimmune disease--the immune complex decoy hypothesis. Nat Clin Pract Rheumatol 2007;3:86-95.

15. Petz LD. Bystander immune cytolysis. Transfus Med Rev 2006; 20:110-40. 